\title{
Réoimes \\ transitoires des groupes réversibles Aspects techniques et économiques
}

\author{
par Mme F. Blanchon, J. Planchard \\ Direction des Etudes et Recherches, E.D.F., Clamart \\ et D. Simonnot \\ Région d'Equipement Hydraulique Nord, E.D.F., Plobsheim
}

Dès le stade du projet d'un aménagement de transfert d'énergie, les effets principaux des régimes transitoires, normaux ou accidentels, auxquels sont voués les groupes hydroélectriques réversibles (survitesses, variations extrêmes de la pression, traversée de plages de fonctionnement critique) peuvent avoir une importance et un rôle déterminants dans la conception de l'aménagement (choix de l'emplacement de l'usine, adoption ou non d'une cheminée d'équilibre) et sur quelques dimensionnements principaux (diamètres d'adductions, moments d'inertie des rotors et encombrement des machines). Comme il y a interdépendance entre ces dimensionnements de base et les effets en question, la recherche d'une solution par approches successives peut s'imposer si, par exemple, surpressions, survitesses, ou diamètres d'adductions, ne peuvent être contenus dans des limites techniquement acceptables, ou si les meilleures conditions économiques ne sont pas obtenues.

\section{Temps de lancer des conduites haute pression}

Les conséquences techniques et économiques des régimes transitoires sont toujours défavorables et trouvent leur origine dans la nécessité pratique de devoir faire varier - dans certains cas, le plus rapidement possible - la force vive de l'eau contenue dans les adductions.

Or, la justification économique des stations de transfert d'énergie est recherchée, entre autres, dans des puissances unitaires très élevées (dépassant $400 \mathrm{MW}$ dans des projets en cours), en tous cas, dans des adductions rapides, de grands diamètres, et caractérisées de ce fait par des temps de lancer $\mathrm{T}_{c}\left({ }^{*}\right)$ élevés.

Dans la gamme des grandes dimensions $\left(\mathcal{O}>10^{7}\right)$, avec des rugosités relatives qui deviennent très faibles (les peintures modernes - époxy ou polyuréthane - permettent d'approcher la perfection et les pertes minimales, caracté-

(*) Voir notations in-fine. ristiques des tuyaux lisses) il est possible, par exemple, de concentrer deux adductions en une seule, avec une augmentation de la vitesse de l'eau de l'ordre de $15 \%$, et ceci sans augmentation des pertes de charge. Le gain sur les quantités totales (volumes excavés, poids des blindages) diminuera dans la même proportion, et le devis de l'adduction encore davantage.

Pour illustrer ce facteur primordial qu'est la force vive de l'eau dans les conduites, on a représenté sur la figure 1 le temps de lancer des adductions haute pression de quelques sites de transfert d'énergie.

Le cas exceptionnel du tronçon commun de Cornwall, qui semble atteindre les limites en la matière, mérite une présentation particulière :

- longueur : $2832 \mathrm{~m}$;

— diamètre : $12,2 \mathrm{~m}$;

- alimentation commune de huit groupes de $250 \mathrm{MW}$.

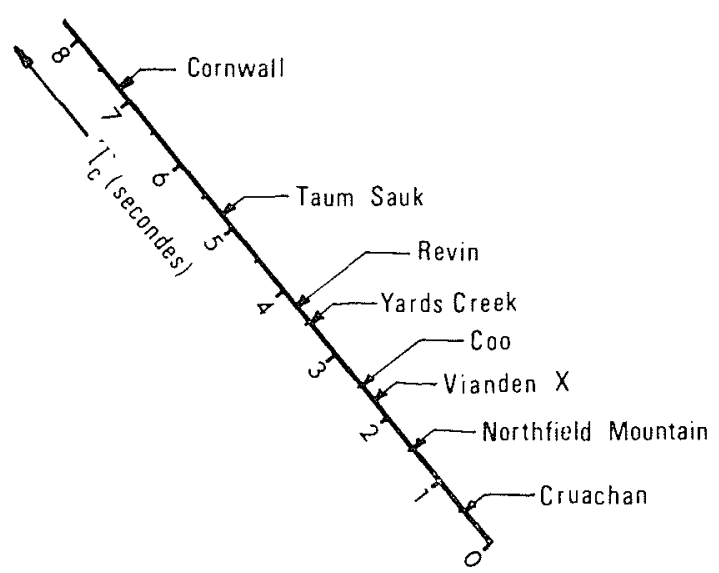

1/ Temps de lancer de quelques adductions haute pression. 


\section{F. BLANCHON, J. PLANCHARD et D. SIMONNOT}

Ce tronçon commun est suivi de rameaux individuels de $600 \mathrm{~m}$ de longueur, parcourus à la vitesse de $11,6 \mathrm{~m} / \mathrm{s}$.

L'économie procurée par la concentration des conduites a des contreparties qui peuvent en dicter les limites:

- augmentation corrélative de l'inertie des machines, sous peine de voir augmenter les coups de bélier, et, par voie de conséquence, la longueur, l'épaisseur et le prix des blindages;

- nécessité de bifurcations, d'autant plus délicates à établir qu'elles sont de grandes dimensions, et qu'elles doivent fonctionner en réversibilité.

\section{Coupure du débit turbine}

A. - Une turbine-pompe possède moins d'aptitudes qu'une Francis équivalente à couper le débit turbiné. Elle en souffre aussi davantage. Ceci tient à plusieurs causes fondamentales, issues de la nécessité de devoir fonctionner en pompe.

$1^{\circ}$ La forme de la machine lui donne la possibilité réelle de pomper dans chaque sens de rotation.

Les caractéristiques débit-vitesse du «quadrant» turbine (chacune correspond à une ouverture du distributeur) tombent verticalement vers le quadrant «pompe à rotation inversée 》 (fig. 2).

A la survitesse où se produit cette inversion, la roue coupe brutalement le débit (ce qui augmente le coup de bélier), et peut même l'inverser (ce qui l'accroît encore).

$2^{\circ}$ Les impératifs du fonctionnement en pompe (rendement et cavitation) obligent à choisir une vitesse qui situe le fonctionnement en turbine entre la vitesse de rendement optimum et celle de l'emballement (à $70 \%$ de celle-ci environ). De ce fait, au cours d'une coupure de débit, le régime d'emballement est à peu près inévitable.

$3^{\circ}$ Le fonctionnement pendant le régime transitoire est caractérisé par une désorganisation notable de l'entrée de l'eau dans la roue: la vitesse relative par rapport aux aubages prend des valeurs importantes du fait de la survitesse de la machine, et perd sa bonne orientation.

Il en résulte des décollements, et le risque de tout un cortège des phénomènes parasites, inaccessibles au calcul:

- hystérésis et instabilité des caractéristiques, engendrant un régime pulsant à la fréquence propre de la conduite;

- mise en «push-pull » de deux machines hydrauliquement couplées par la même adduction, etc.

B. - Le graphique de la figure 3 représente, dans un cas concret, trois possibilités de trajectoires de fonctionnement transitoire d'une pompe-turbine, toutes choses restant égales par ailleurs (adductions, $\mathrm{MD}^{2}, \ldots$ ).

Trajectoire I: fermeture linéaire du distributeur en $60 \mathrm{~s}$.

Trajectoire II : fermeture en deux temps :

$$
\begin{aligned}
& \text { - de } 1 \text { à } 0,75 \text { en } 1 \mathrm{~s} \text {; } \\
& \text { — de } 0,75 \text { à } 0 \text { en } 45 \mathrm{~s} \text {. }
\end{aligned}
$$

Trajectoire III : fermeture en trois temps :

$$
\begin{array}{lll}
\text { - de } 1 & \text { à } 0,625 & \text { en } 1,5 \mathrm{~s} \text {; } \\
\text { - de } 0,625 \text { à } 0,45 & \text { en } 2,1 \mathrm{~s} \text {; } \\
\text { - de } 0,45 & \text { à } 0 & \text { en } 9,2 \mathrm{~s} .
\end{array}
$$

On a porté sur quelques points de ces trajectoires la valeur sommairement calculée de la vitesse relative, et de l'angle de cette vitesse avec les aubages. On voit notamment comment la zone de turbine accélératrice est perturbée, et comment une loi de fermeture de distributeur à plusieurs pentes, très rapide au début de la manœuvre, permet de diminuer substantiellement la vitesse relative et les inconvénients des décollements. (La diminution de la survitesse périphérique qui est à l'origine de ce gain, est aussi très appréciée par le rotor de l'alternateur-moteur.)

On serait tenté d'admettre sans contrôle que la trajectoire I est préférable à la trajectoire II en ce qui concerne le coup de bélier (coupure du débit dans le temps le plus long) Ce serait une erreur.

Avec la fermeture la plus lente, on observe dans toute son ampleur (fig. $4 \mathrm{I}$ ) la coupure du débit par la roue et une pointe correspondante sur la surpression. De plus, l'inversion du débit, qu'on ne peut éviter, accroît la pente de $\mathrm{Q}(t)$, et par conséquent le coup de bélier. Enfin, le groupe atteint une vitesse extrême dépassant très largement la vitesse d'emballement permanent (de 10 , voire $15 \%$ par exemple).

L'expérience montre qu'en réduisant le temps de fermeture, avec une loi de manœuvre adéquate, il peut être possible (fig. 4 II) :

- de contenir la surpression dans les mêmes valeurs que précédemment en «nivelant»- dans une certaine mesure - la courbe de la surpression, dont l'intégrale est, à un facteur constant près, imposée par la variation de débit obtenue:

$$
\int_{0}^{t} \Delta \mathrm{H} d t / \mathrm{T}_{0} \mid \Delta \mathrm{Q}_{0}^{t} \text { est constant }
$$

- de réduire substantiellement la vitesse maximum transitoire.

C. - Le moment d'inertie $\left(\mathrm{MD}^{2} / 4\right)$ et, plus précisément, le temps de lancer du groupe:

$$
\mathrm{T}_{y}=1 / 4 \mathrm{MD}^{2} \Omega^{2} / \mathrm{W}
$$

caractérise le réservoir dans lequel on stocke l'énergie mécanique développée par la turbine pendant la durée qu'il faut donner à la coupure du débit pour éviter une surpression excessive.

A ce titre, le temps de lancer conditionne directement la survitesse maximum prise par le groupe et, par suite, le calcul de nombre d'organes de la machine.

Mais, dans le cas des turbines dotées de caractéristiques telles que représenté figure 2 , le $\mathrm{MD}^{2}$ a un rôle plus important, d'incidence écoromique considérable : par l'atténuation qu'il inflige à la survitesse transitoire, il diminue l'effet de coupure du débit par la roue, et réduit d'autant la surpression. Il y a donc une sorte d'équivalence, vis-à-vis des surpressions, entre un temps de lancer d'adduction court et un $\mathrm{MD}^{2}$ élevé (on rappelle qu'une équivalence analogue existe vis-à-vis de la stabilité sur réseau séparé). C'est la puissance apparente et la vitesse qui fixent le volume caractéristique $\left(d^{2} 1\right)$ du rotor de l'alternateur-moteur.

La possibilité d'exécuter, pour le même volume, plusieurs valeurs du moment d'inertie (grand diamètre pour une faible hauteur, ou vice-versa) dépend de plusieurs facteurs :

- vitesse périphérique maximum admissible;

- modification corrélative du nombre d'encoches et, partant, du schéma d'enroulement;

- prix de revient. 
Il est important de souligner que, du seul point de vue technique, certaines vitesses se prêtent mieux que d'autres à cette possibilité de faire varier, sans trop de concessions économiques, le $\mathrm{MD}^{2}$ de la machine (celles, en particulier, pour lesquelles le nombre de paires de pôles est multiple du nombre de voies d'enroulement). Cette notion doit rester présente à l'esprit au moment du choix du nombre de groupes.

La figure 5 donne à titre indicatif, en fonction du $\mathrm{MD}^{2}$, la variation relative du prix d'un alternateur-moteur et celle $\mathrm{du}$ moteur asynchrone (poney) devant assurer la mise en vitesse dans le sens de rotation du pompage. Le prix marginal de l'inertie d'une machine s'étend à bien d'autres rubriques (volume d'implantation, accès, ponts roulants, etc.).

D. - La réduction de longueur de l'adduction haute pression, obtenue en rapprochant l'usine de la prise d'eau supérieure, est bien entendu très bénéfique du point de vue des survitesses et des surpressions.

Lorsqu'elle est poussée au maximum (usine en pied de descenderie), elle a pour effet favorable de réduire la longueur et l'épaisseur des blindages, d'autoriser l'utilisation de machines à faible inertie et, éventuellement, d'un procédé de lancement simple et économique (démarrage en asynchrone si l'impédance du réseau le permet).

Mais elle déplace les problèmes et alourdit les investissements sur d'autres rubriques :

- allongement corrélatif des accès;

- difficultés accrues pour l'exhaure et la sécurité contre l'inondation;

- problème des raccordements au réseau (transformateurs en caverne et liaisons par câbles T.H.T, inévitables);

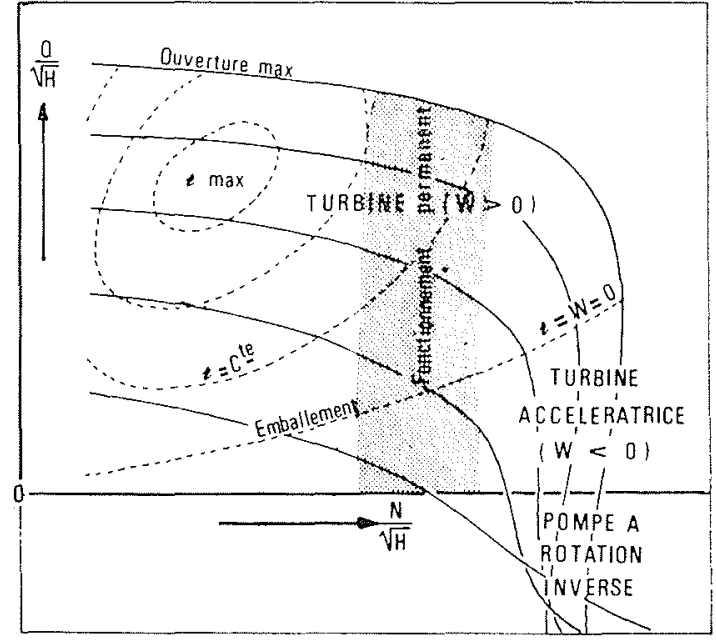

2/

2/ Régimes de fonctionnement pendant la coupure du débit turbiné

3/ Coupure du débit turbiné. Influence de la loi de manœuvre du distributeur.

4/ Allure des coups de bélier obtenus par les lois de manœuvre de la figure 3.

5/ Prix marginal de l'inertie, concernant la machine elle-même.

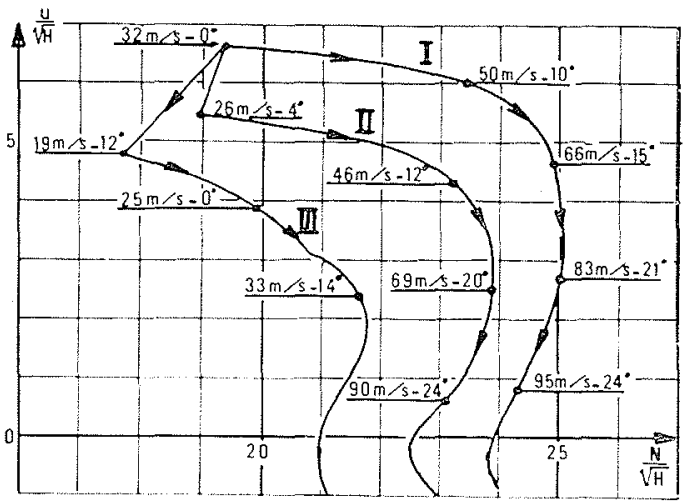

3/
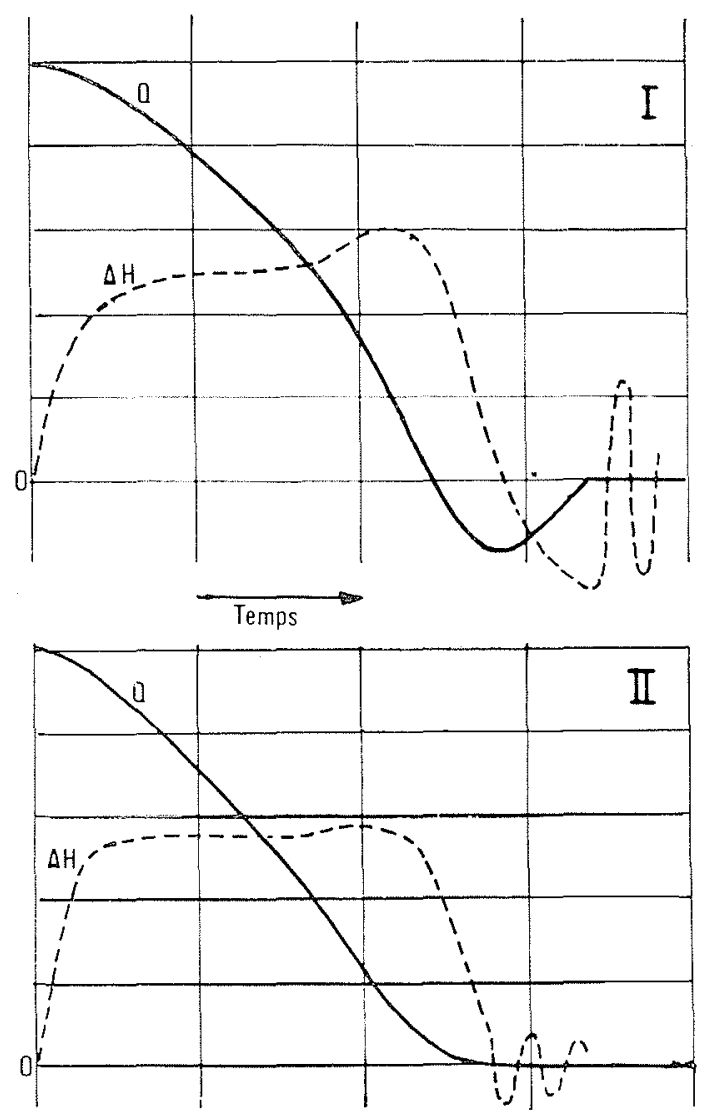

4/

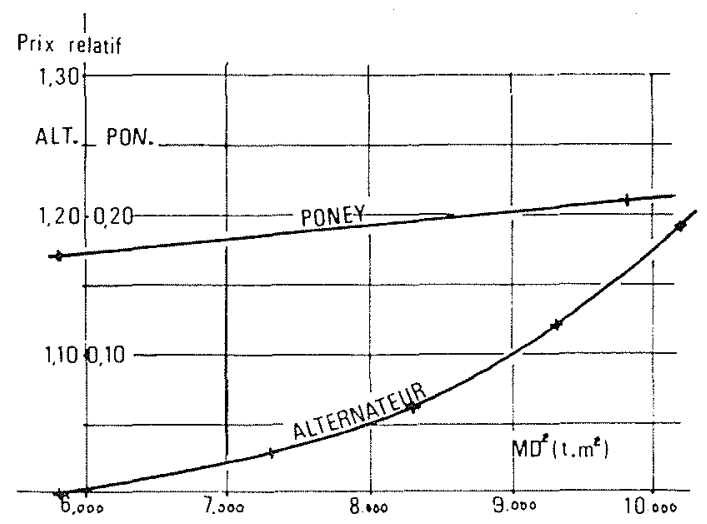

5) 


\section{F. BLANCHON, J. PLANCHARD ef D. SIMONNOT}

- importance considérable de l'adduction basse pression, qui ne peut tolérer que des vitesses d'eau modérées;

- nécessité d'une cheminée d'équilibre sur cette adduction.

La cheminée d'équilibre, à elle seule, pose au projeteur toute une série de problèmes:

$1^{\circ}$ Son dimensionnement, et en particulier celui de ses chambres d'expansion, est à étudier en vue de limiter dans l'adduction basse pression:

- la surpression à l'arrêt du pompage ou au dévirage et, accessoirement (si l'on désire «travailler» particulièrement la promptitude des manœuvres de la machine), à la prise du débit en turbinage;

- la dépression à la coupure du débit turbiné et à la prise du débit en pompe.

Cette étude ne doit pas oublier les cas accidentels qui peuvent survenir, tant peuvent être graves les conséquences d'un dépassement imprévu (absence d'un déversoir, danger d'une cavitation généralisée).

$2^{\circ}$ Pour ajuster efficacement son amortissement, il est indiqué de choisir un schéma fournissant en suffisance les paramètres ou degrés de liberté nécessaires (par exemple, cheminée double, caractérisée par deux pseudo-périodes convenablement différenciées, et dotées d'organes d'amortissements tels que diaphragmes et déversoirs).

$3^{\circ}$ La stabilité est à examiner en fonction du mode de fonctionnement recherché.

L'effet déstabilisant de l'inertie de l'eau dans l'adduction haute pression - inertie qui sera souvent, et malgré tout, importante dans les aménagements de transfert d'énergie ne peut plus être négligé, comme il est fait dans la procédure d'établissement de la section de Thoma, si la machine doit être asservie en puissance. Bien entendu, il conviendra d'introduire en contrepartie dans l'étude l'effet stabilisateur de la constante de temps du réglage, dont la valeur fait l'objet de prescriptions par E.D.F. dans le cahier des charges des machines. La stabilité d'une installation en projet est assez aisée à vérifier par l'application numérique d'un critère analytique classique (critère de Routh par exemple). En revanche, les conditions de la stabilite sont trop complexes pour que des orientations simples - comme, par exemple, la formule de Thoma - puissent être dégagées.

$4^{\circ}$ Les conséquences éventuelles de certains phénomènes secondaires peuvent en justifier un examen approfondi :

- excitation des résonances de la cheminée d'équilibre par certains spectres de l'ordre de téléréglage (les remèdes à opposer ayant toujours pour effet de relâcher l'asservissement);

- introduction de la fonction de transfert résultant de l'inertie de l'eau dans la cheminée, la hauteur de la colonne liquide pouvant atteindre l'ordre de grandeur de $50 \mathrm{~m}$ du fait de «l'enfoncement $»$ requis par les turbinespompes.

$5^{\circ}$ La section de surface libre de la cheminée d'équilibre, nécessaire pour la stabilité, doit être excavée sur toute la hauteur de marnage du bassin inférieur. Il peut s'ensuivre des difficultés de réalisation et, en tout cas, d'importantes conséquences sur le coût.

Les problèmes évoqués ci-dessus enlèveraient aux cheminées d'équilibre, s'il en était besoin, leur image de remède universel. Et, quels que soient les soins apportés à leur établissement, on doit constater que ces organes ne peuvent qu'entamer la perfection du service rendu par les machines réversibles, spécialement sur le plan de leur asservissement.

\section{Perte du couple moteur en pompage}

La perte du couple moteur est suivie par une baisse rapide, puis une inversion du débit, qui engendrent une surpression dans l'aspirateur de la machine, et une dépression transitoire dans l'adduction haute pression. Parallèlement (mais avec le retard dû à l'inertie du groupe) le groupe ralentit, puis dévire, la fin de la manœuvre étant une coupure de débit turbiné.

A. - La dépression transitoire, dans l'adduction haute pression, doit être examinée au regard de la tenue des blindages aux sous-pressions.

Mais son effet le plus marqué provient de son gradient le long de la conduite, et plus particulièrement au voisinage de la prise d'eau supérieure. Ce gradient peut être assez élevé pour conduire à une cavitation si l'enfoncement n'est pas suffisamment rapide.

Ce phénomène peut ainsi éliminer toute solution de conduite aérienne (tracé A sur la figure 6) au profit d'un puits ou d'une descenderie (tracés B ou C). Il est clair que l'installation de vannes de tête sur des conduites telles que $\mathrm{B}$ ou $\mathrm{C}$ est difficile et très onéreuse : elle est rarement pratiquée.

B. - Le régime infligé à la turbine-pompe dans le quadrant pompe-frein (vitesse en pompe, débit en turbine) est redoutable. La vitesse relative moyenne de l'eau à l'entrée de la roue, représentée figure 7 , rend inutile tout commentaire.

La fermeture rapide du distributeur procure, ici également, des avantages certains :

- diminution importante du débit et de la puissance dissipée dans le quadrant «pompe-frein »;

- réduction de la sous-pression transitoire;

- suppression du dévirage.

La figure 7 représente concrètement l'évolution du régime d'une machine selon la loi de mancuvre adoptée. On constate que la loi $\mathrm{n}^{\circ}$ III, exécutée en deux temps, permet la coupure expéditive du débit, avec les variations de pression les plus fajbles. Elle abandonne la machine au barbotage, à une vitesse voisine de $60 \%$ de la vitesse nominale et qui correspond à un ralentissement de longue durée. Mais en allongeant convenablement le second temps de la manœuvre, on peut à la fois limiter le débit inverse à une valeur acceptable, et réaliser un freinage naturel, efficace, et très appréciable à plusieurs égards (simplification considérable des séquences d'automatisme; diminution des contraintes infligées au pivot et à la couronne de freinage).

\section{Autres régimes transitoires}

Par comparaison avec les cas évoqués ci-dessus, les autres régimes transitoires normaux ne procurent pas de difficultés importantes et n'exigent guère d'investissements particuliers :

- la vitesse de prise de débit en turbinage (et les variations de pression qui en découlent) peut toujours être réglée 
à une valeur inférieure à celle qui résulte de la perte de couple en pompage, par simple allongement de la vitesse d'ouverture, et sans que la promptitude de la machine cesse d'être convenable;

- la prise de débit en pompage est surtout caractérisée par la dépression à l'aspiration, et peut conditionner directement la spécification des blindages (garanties de tenues aux sous-pressions) ainsi que le dimensionnement de l'éventuelle cheminée d'équilibre. La vitesse d'ouverture du distributeur, au début de la mancuvre, est le paramètre principal en cette matière.

Quelques régimes transitoires, redoutés, de caractère purement accidentel, peuvent également imposer une étude, et conditionner certains dimensionnements. Leur nature dépend de nombreux facteurs (souvent propres à l'installation), des différents stades de sécurité qu'on se fixe, etc., de sorte qu'il n'est pas question d'évoquer ici un modèle en cette matière. A titre d'exemple, nous citons ci-après deux études particulières effectuées dans le cadre du projet de Revin :

$1^{\circ}$ Recherche de la loi de fermeture des robinets sphériques permettant la coupure du débit de gueule bée maximum d'un rameau haute pression, avec les deux conditions suivantes, qui concernent la sécurité publique:

- surpression n'excédant pas les valeurs contractuelles normales;

- volume d'eau cumulé inférieur à la capacité que présente la caverne de l'usine au niveau de l'exutoire naturel (galerie d'accès).

$2^{\circ}$ Recherche de la vitesse maximum admissible pour la fermeture du distributeur des turbines-pompes. Cette étude a pour but d'éviter les conséquences désastreuses qui résulteraient d'une défaillance de l'organe limitant la vitesse de fermeture dans la dernière phase d'une fermeture en deux temps. Ces conséquences peuvent être:

- des déformations plastiques consécutives aux surpressions;

- un choc catastrophique par marteau d'eau si la surpression atteint $100 \%$ (processus représenté figure 8).

Au regard de ces problèmes, une adduction ramifiée est très favorable si la défaillance n'est raisonnablement envisagée que sur une seule machine à la fois.

Il n'est pas sans intérêt de constater qu'avec la surpression maximum prévue pour des régimes transitoires normaux (40\%) et le coefficient de sécurité adopté pour les conduites (contrainte maximum normale ne dépassant pas la moitié de la limite élastique), la cavitation préalable au choc par marteau d'eau apparaît avant les surpressions excessives quand on raccourcit trop le temps de fermeture linéaire.

\section{Exemple d'influence des régimes transitoires sur un bilan économique}

Il est tout naturel qu'à certains stades de l'établissement d'un projet, des comparaisons économiques sojent introduites pour aider à lever certaines options. Il ne serait pas sérieux, en revanche, d'orienter les décisions en fonction de ces seuls critères: il n'est guère possible d'une part d'aller avec exactitude jusqu'au bout de ces comparaisons; d'autre

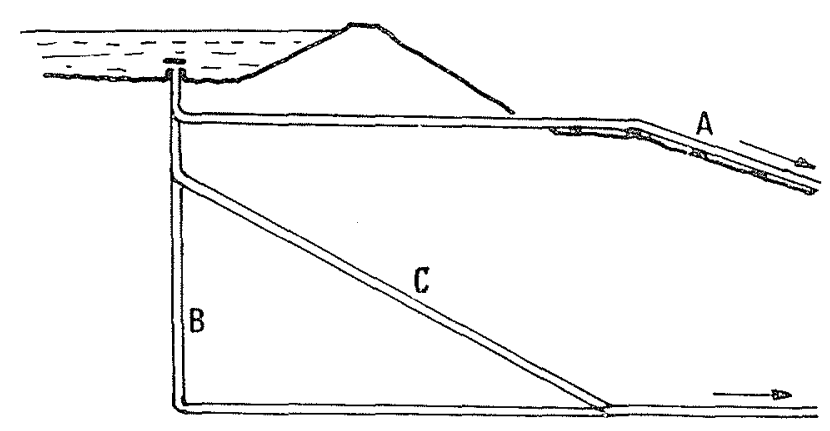

6/ Tracés dadduction haute pression.

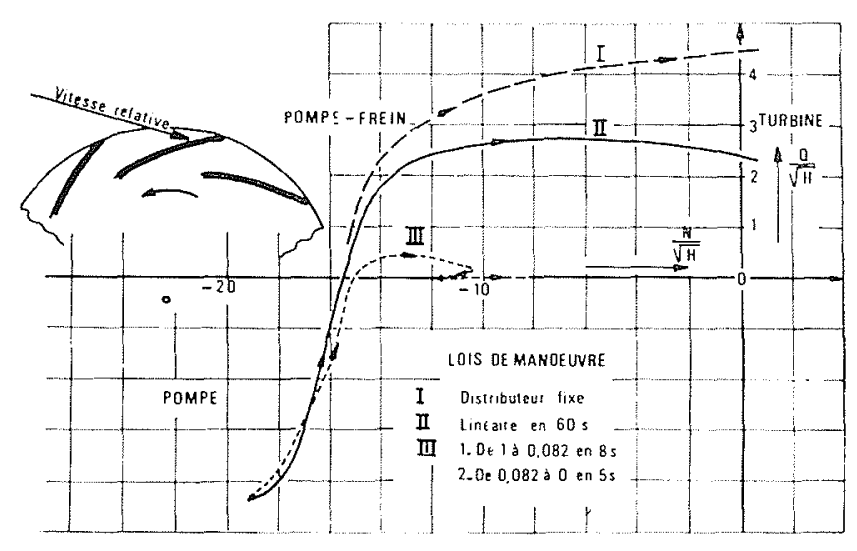

7/ Perte du couple moteur en pompage.

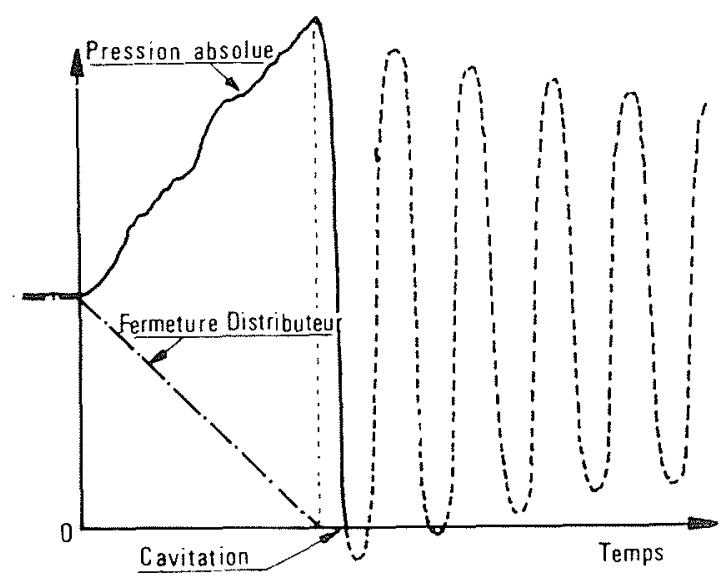

8/ Vitesse de fermeture maximum admissible. 


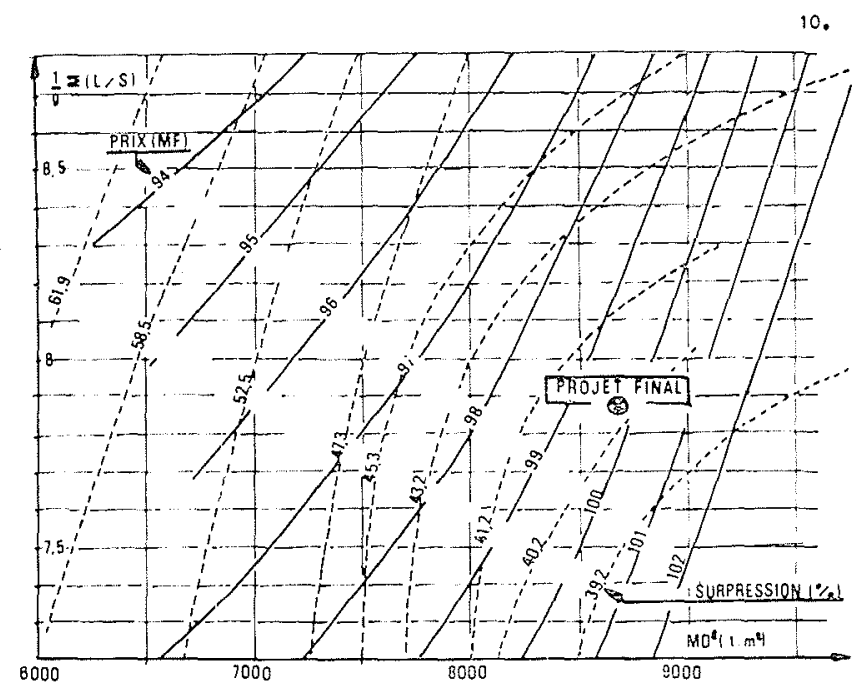

9/ Infuence économique des adductions et du $\mathrm{MD}^{2}$ (Revin).

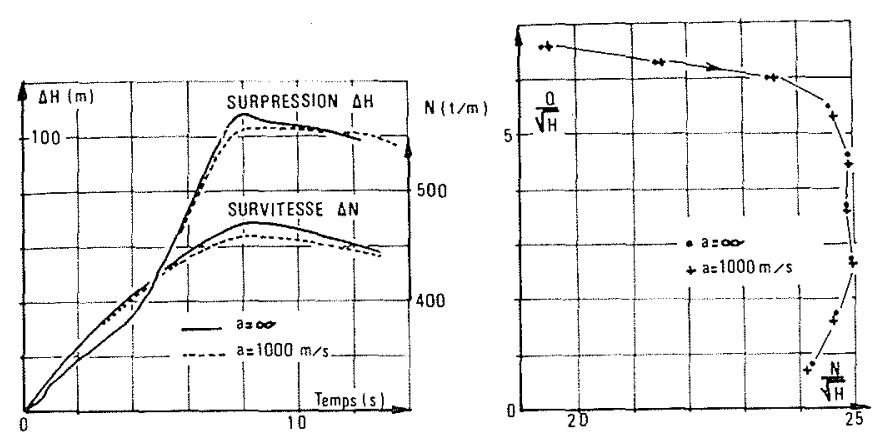

10/ Comparaison des régimes transitoires calculés, selon la célérité $a$.

part, d'éviter certaines incertitudes et certaines contraintes majeures, telles que celles qui concernent les possibilités ou les délais de réalisation, les jugements sur la sécurité, etc.

C'est à l'abri de ces restrictions, que nous donnons, figure 9, le résultat graphique d'une étude économique partielle se rapportant au projet de Revin. On a représenté :

- les surpressions maximales (coupure du débit maximum turbiné) en fonction du $\mathrm{MD}^{2}$ et du temps de lancer de l'adduction;

- la variation du prix global de:

- l'alternateur (dépend du $\mathrm{MD}^{2}$ );

- moteur de lancement (dépend du MD²);

- pertes électriques (dépendant de l'alternateur, donc $\mathrm{du} \mathrm{MD}^{2}$ );

- l'adduction, y compris blindages (dépend des diamètres choisis et de la surpression obtenue);

- les pertes de charge (dépend des diamètres choisis).

A. - On constate que le projet choisi ne paraît pas conforme au meilleur choix économique: le prix global diminue quand la surpression acceptée croît.
En fait, on a limité la surpression à environ $40 \%$ :

- par refus, entre autres, des difficultés que présente, audelà de cette valeur, la construction des blindages haute pression (nécessité du recuit des viroles après cintrage);

- en raison des inconvénients de l'accroissement corrélatif de la survitesse maximum des groupes réversibles.

B. -- Nombre d'éléments, d'incidence économique secondaire, n'ont pas été pris en compte. Par exemple:

- influence de la surpression sur le prix des bâches;

— influence de la survitesse sur le prix de l'alternateur et du pivot;

- variations de prix sur les matériels d'alimentation des moteurs poneys;

- toutes les conséquences économiques des variations de dimensionnement des machines (prix de la caverne, des ponts roulants, etc.).

C. - Il n'est pas besoin de souligner que l'établissement du graphique de la figure 9 a exigé la réalisation méthodique d'un grand nombre d'《épures» (de l'ordre d'une centaine en l'occurrence). Les surpressions calculées dépendent des lois de manœuvre adoptées; pour obtenir des résultats homogènes, on a recherché, pour chaque épure, la loi de manceuvre optimale, et non linéaire, permettant d'obtenir le coup de bélier minimum.

Cette recherche a été exécutée dans le cas simple d'une célérité infinie, ce qui a permis d'effectuer rapidement les «épures» au moyen d'une petite calculatrice à programmation directe. La figure 10 témoigne, par un exemple de comparaison avec un calcul rigoureux, que la précision de cette méthode simplifiée est bien suffisante en pratique: on remarque en particulier que les trajectoires de fonctionnement sont exactement confondues.

Automatisation du calcul des régimes transitoires

Dans le cadre d'un projet de station de transfert d'énergie, le calcul des régimes transitoires est finalement complexe et volumineux et peut être, pourtant, déterminant.

Le calcul, aussi précis que possible, par les méthodes traditionnelles se heurte à des difficultés pratiques considérables:

- la réversibilité exige l'étude de plusieurs cas principaux;

- la sévérité des coups de bélier peut contraindre à rechercher une loi de manœuvre optimum (à double pente par exemple);

- les adductions ramifiées et les cheminées d'équilibre rendent les épures très complexes;

- l'évolution des phénomènes ne peut se calculer qu'au moyen d'une équation différentielle annexe (masses tournantes) suivie, pas à pas, d'une transposition des caractéristiques de la machine;

- la célérité n'étant jamais prévue à coup sûr, certains calculs peuvent devoir être menés avec plusieurs valeurs de cette variable.

De surcroît, ces études doivent être exécutées très rapidement si tant est que les projets sont généralement très 


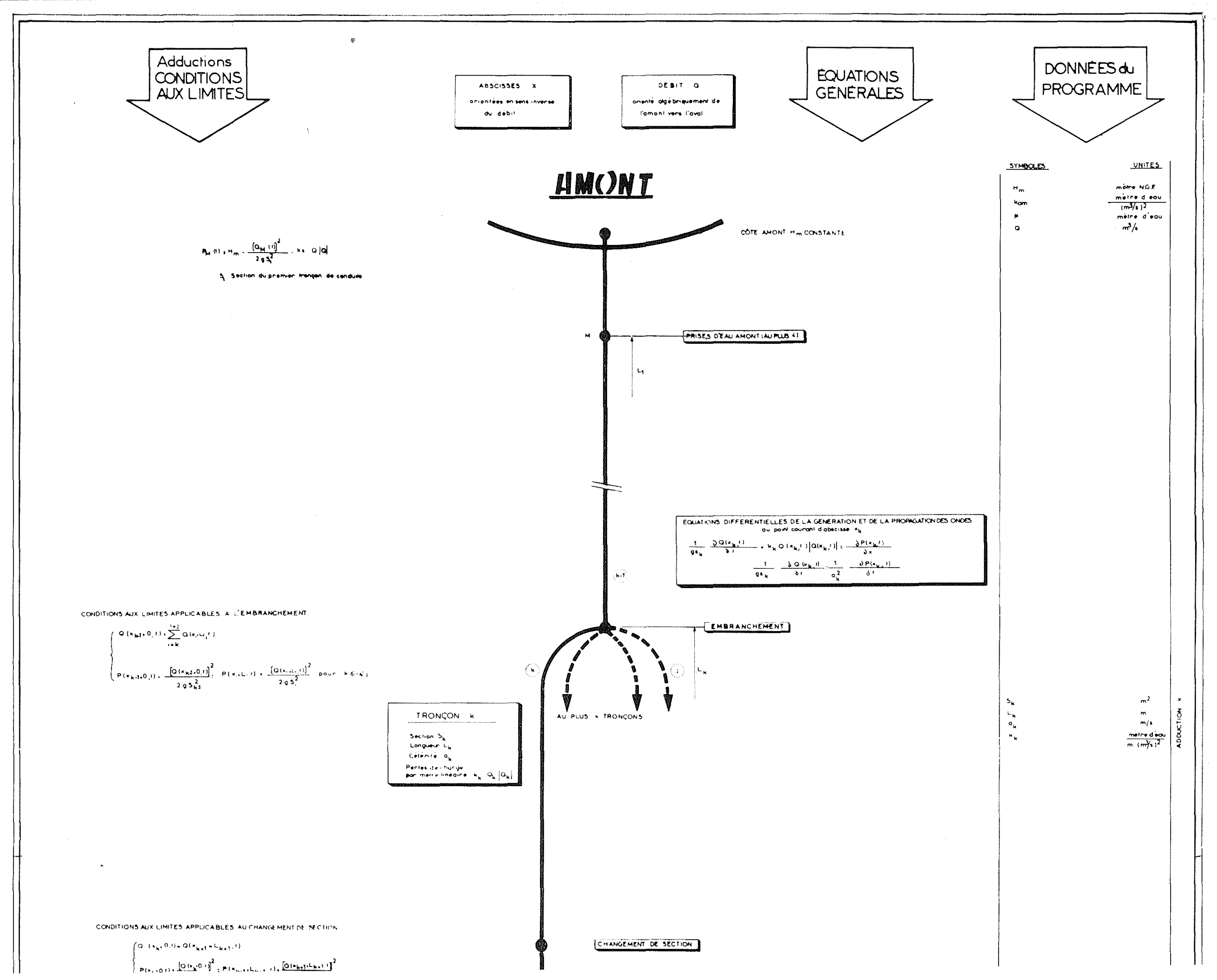


avancés quand les caractéristiques du modèle de la machine, nécessaires aux calculs, sont connues.

L'ensemble de ces considérations a conduit E.D.F. à mettre au point la programmation des calculs pour leur exécution par ordinateur (programme 1316 du Département T.I.E.M. de la Direction des Etudes et Recherches)

A. - Le cadre accessible à ce programme est celui d'un ensemble représenté par le plan Re 550 a, et comportant de un à quatre groupes réversibles, quel que soit le schéma de ramification des adductions.

Des cheminées d'équilibre «différentielles 》sont facultatives, et possibles, en haute comme en basse pression.

$B$. - Les relations physiques traitées par le calcul sont indiquées sur le plan $\operatorname{Re} 550 \mathrm{a}$. On se contentera ici d'apporter les précisions suivantes:

\section{a) Prises d'eau et convergents.}

Aux changements de section de la veine liquide, on a introduit la variation résultante de la pression, dans les conditions idéales d'un écoulement sans perte (variation de la pression dynamique seule).

Pour les prises d'eau, on a ajouté un terme représentant la perte de charge, qui peut prendre une importance particulière si la prise comporte une grille. (Par simplification pour le programme, la perte de charge a été considérée indépendante du sens de l'écoulement).

Aux changements de section, la perte de charge locale n'a pas été prévue au calcul : il faudra l'introduire linéiquement dans l'adduction adjacente (ce qui est, dans une certaine mesure, conforme à la réalité),

\section{b) Tronçon courant.}

Dans un tronçon courant de section uniforme, la pression et le débit sont reliés par deux équations introduisant l'élasticité et la masse, et donnant lieu de ce fait aux phénomènes de propagation, caractérisés par la célérité. Les pertes de charge ont été introduites par le biais d'un coefficient qui laisse au phénomène sa répartition linéique réelle. C'est, sur ce point, une différence essentielle entre le programme 1316 et la méthode graphique (épure de Bergeron).

\section{c) Embranchements.}

Aux embranchements, on a introduit la variation de la pression dynamique consécutive aux éventuels changements de section. Mais on a renoncé à introduire les pertes de charge car les critères qui les caractérisent sont très complexes, et rarement connus en détail au moment de l'exécution des calculs. Il convient donc de répartir « grosso modo» la perte de charge d'embranchement sur les tronçons adjacents. Une telle approximation est d'ailleurs sans importance sensible sur les résultats du calcul.

\section{d) Pompes-turbines.}

En matière de calculs, la pompe-turbine $n^{\prime \prime} i$ est essentiellement une relation entre les grandeurs suivantes:

- l'ouverture $\mathrm{O}_{i}$;

- la hauteur nette $\mathrm{H}_{n i}$ (chute ou refoulement);

- la vitesse $\mathrm{N}_{i}$;

- le couple $\mathrm{C}_{i}$;

- le débit $Q_{i}$.

(Les deux dernières sont en quelque sorte une conséquence des trois premières.)

En pratique, on entre les caractéristiques des pompes- turbines dans le programme sous la forme de deux familles de courbes, très habituelles chez les hydrauliciens:

$1^{\circ}$ Le débit réduit $\left(\mathrm{Q}_{1}=\mathrm{Q} / \sqrt{\mathrm{H}_{n}}\right)$ en fonction de la vitesse réduite $\left(\mathrm{N}_{1}=\mathrm{N} / \sqrt{\mathrm{H}_{n}}\right)$, l'ouverture étant un paramètre (une courbe $Q_{1} / N_{1}$ par ouverture).

$2^{\circ}$ Le couple réduit $\left(\mathrm{C}_{1}=\mathrm{C} / \mathrm{H}_{n}\right)$ en fonction de la vitesse réduite, l'ouverture étant un paramètre (une courbe $\mathrm{C}_{1} / \mathrm{N}_{1}$ par ouverture).

Il faut faire toutefois deux remarques importantes.

La première est que les courbes à utiliser sont relatives à un fonctionnement de machine non affecté par la cavitation. (On a, bien entendu, renoncé à introduire le paramètre correspondant, sous peine d'une énorme mobilisation de mémoires, et d'une grande complication d'emploi du programme).

La seconde remarque concerne l'allure des courbes du débit réduit et du couple réduit. Une relation univoque entre $Q_{1}$ et $N_{1}$ d'une part, entre $C_{1}$ et $N_{1}$ d'autre part, convient parfaitement au programme. S'il n'en est pas ainsi dans la zone des vitesses en turbinage, et si une valeur de $\mathrm{N}_{1}$ conduit au maximum à trois valeurs de l'ordonnée, le programme est encore utilisable.

\section{e) Boucle de vitesse.}

La vitesse de la machine résulte directement du couple qu'elle produit, et du couple résistant qu'on lui impose.

Ce dernier comporte deux ensembles:

- Le couple d'inertie et le couple résistant de la machine elle-même (réseau « minimal »);

- les mêmes éléments relatifs au réseau alimenté (réseau « extérieur »).

Les paramètres choisis pour le réglage sont ceux d'un régulateur accélérotachymétrique simple: promptitude, dosage accélérométrique et statisme. (Les paramètres d'un régulateur à asservissement temporaire sont assimilables aux précédents avec une précision suffisante eu égard à l'ambition de ce programme.)

Pour permettre l'étude du comportement des machines lors des grands régimes transitoires, on a donné à la promptitude les butées qui entrent normalement en jeu dans la réalité, et qui découlent de la limitation de la vitesse de manœuvre du distributeur.

On a donc donné un double écrêtement à cette vitesse dans le sens de l'ouverture, et un double écrêtement dans le sens de la fermeture. On simule ainsi les lois d'ouverture et de fermeture à double pente qui seront souvent néces. saires sur les groupes réversibles.

On n'a pas cherché à représenter la boucle - assez souvent utilisée maintenant - de l'asservissement à la puissance : les études correspondantes n'apportent aucun enseignement qui soit de nature à influencer un projet. (Les perfectionnements apportés par cet asservissement concer nent principalement la précision de la participation au réglage primaire et secondaire. En revanche, la boucle correspondante est de nature à éveiller des instabilités, surtout quand il existe une cheminée d'équilibre.)

\section{f) Cheminée d'équilibre.}

Il n'est guère contesté à présent que les cheminées permettant les mises au point les plus aisées sont celles qui donnent lieu à deux périodes ou pseudo-périodes, et qui sont dotées d'un organe brisant l'énergie.

Les caractéristiques des cheminées introduites dans le programme sont, dans cet esprit, celles des cheminées diffé- 

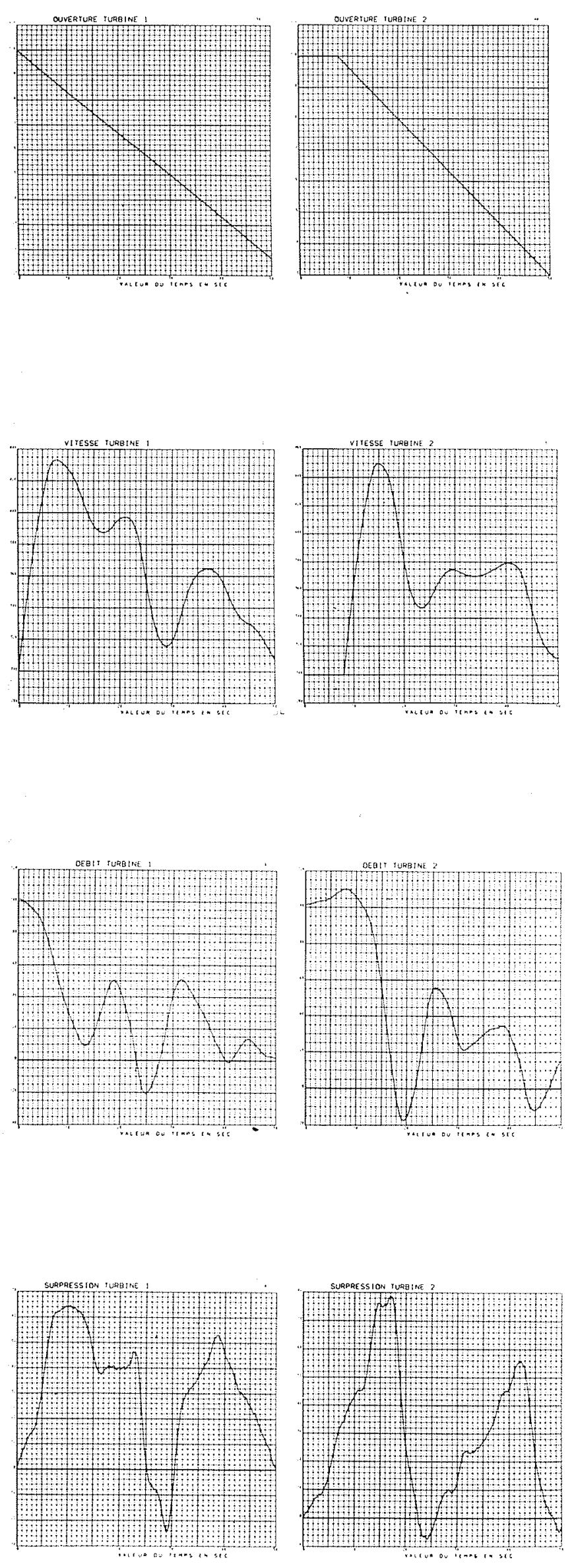

11/ Graphiques fournis par l'ordinateur. rentielles comportant un puits non amorti en parallèle avec un puits (de plus grande section) équipé d'un diaphragme.

Les grandes oscillations apparaissent dans le premier, et s'amortissent soit au passage du débit de vase communicant à travers le diaphragme, soit, à la limite, par déversement.

On a poussé le réalisme en admettant une dissymétrie du coefficient d'absorption dans le diaphragme, et en permettant l'expansion dans des chambres.

C. - La méthode de calcul utilisée pour traiter les phénomènes en propagation repose essentiellement sur la discrétisation, en temps et en espace, des équations élémentaires reliant le débit $\mathrm{Q}$ et la pression $\mathrm{P}$ le long de chaque tronçon:

$$
\begin{gathered}
\frac{1}{g \mathrm{~S}} \frac{\partial \mathrm{Q}(x, t)}{\partial t}=\frac{\partial \mathrm{P}(x, t)}{\partial x}-k \mathrm{Q}(x, t)|\mathrm{Q}(x, t)| \\
\frac{1}{a^{2}} \frac{\partial \mathrm{P}(x, t)}{\partial t}=\frac{1}{\mathrm{gS}} \frac{\partial \mathrm{Q}(x, t)}{\partial x}
\end{gathered}
$$

En remplaçant les termes différentiels par des différences finies, on obtient :

$$
\begin{aligned}
\frac{1}{g \mathrm{~S}} \frac{\mathrm{Q}(x, t+\Delta t)-\mathrm{Q}(x, t)}{\Delta t} & =\frac{\mathrm{P}(x+\Delta x, t)-\mathrm{P}(x-\Delta x, t)}{2 \Delta x} \\
& -k \mathrm{Q}(x, t)|\mathrm{Q}(x, t)|
\end{aligned}
$$

et une expression similaire pour la seconde équation, les débits étant pris à l'instant $t+\Delta t$.

Ces formules permettent de calculer les débits et pressions au temps $t+\Delta t$, connaissant leurs valeurs à l'instant $t$. On effectue ainsi un calcul pas à pas à partir du régime initial.

Des relations particulières sont introduites aux singularités (changements de section, prises d'eau, bifurcations) pour assurer la conservation du débit et de la pression totale.

Le programme s'accommode des caractéristiques multivoques (à une vitesse réduite $N_{1}$ correspondent plusieurs valeurs du débit ou du couple réduits $Q_{1}, C_{1}$ ): une procédure automatique de recherche du point de fonctionnement a dû être mise au point pour cela.

Il s'accommode également de n'importe quel schéma d'installation dont la configuration peut être décrite sur cartes « données ». Il comporte de nombreux sous-programmes relatifs à des éléments particuliers (tronçons de conduites, bifurcations, turbines-pompes, cheminée, régulateur, etc.) que le programme relie automatiquement selon le schéma de l'installation, et en fonction des calculs effectués.

Plusieurs régimes transitoires peuvent être ainsi simulés:

- arrêt d'urgence en turbine;

- démarrage en turbine;

- perte du couple moteur en pompe, avec fermeture du distributeur;

- perte du couple moteur en pompe, avec distributeur fixe; - prise du débit en pompe;

- fonctionnement en turbine sur réseau séparé (stabilité);

- fonctionnement en turbine sur réseau infini (réponse au téléréglage).

D. - Les résultats de calcul (débit dans la machine, pressions aux endroits singuliers des adductions, vitesses de rotation) sortent de la machine sous forme de listings, complétés par une impression automatique de diagrammes, comme représenté en réduction figure 11 (fermetures différées de deux turbines-pompes placées en parallèle). 


\section{Symboles utilisés}

$\mathrm{T}_{c}=\frac{\mathrm{LV}}{g \mathrm{H}}:$ temps de lancer d'une conduite, avec :
L: longueur;
$\mathrm{V}$ : vitesse de l'eau;
$g$ : accélération de la pesanteur;
$\mathrm{H}$ : hauteur de chute;
$\mathcal{O}:$ nombre de Reynolds;
$t$ : temps;
Q : débit;
$\mathrm{N}$ : vitesse;
$\mathrm{W}$ : puissance;
$r$ : rendement; moment d'inertie);
$\mathrm{T}_{g}$ : temps de lancer du groupe réversible;
$\Omega$ : vitesse angulaire;
$\mathrm{P}$ : pression;
$x$ : abscisse le long de la conduite;
$\mathrm{S}$ : section de la conduite;
$a$ : célérité.

$\mathrm{MD}^{2}$ : facteur d'inertie de la machine (quadruple du

\section{Discussion}

Président : M. J. N. PI.ICHON

M. lc Président remercie le conférencier et félicite les auteurs de la communication présentée; à l'occasion de l'étude particulière de l'usine de Revin, ils ont procédé à une investigation de caractère plus général et mis au point un programme de calcul des surpressions en régime transitoire qui s'applique à de nombreux cas. Ce point est très important car de tels calculs sont souvent indispensables au projeteur pour procéder à certains choix fondamentaux : emplacement optimum de l'usine, tracé des galeries d'adduction, etc. Il sera, d'ailleurs, d'un grand intérêt de comparer les résultats des calculs ci-dessus à ceux résultant des mesures faites sur l'usine au moment de sa mise en service.

Avant d'ouvrir la discussion, $M$. le Président demande à $M$. PLANCHARD :

A l'examen de la figure 9, il semble que les dispositions adoptées dans le projet définitif ne correspondent pas au minimum - pourtant généralement « très plat »- du prix total résultant de votre étude économique. Pourquoi?

M. Simonnot répond en s'aidant de la projection de la figure 9; ce graphique, dit-il, ne constitue qu'une partie de l'étude d'optimisation, laquelle ne comporte pas que des critères économiques; ainsi, le choix d'une surpression de l'ordre de $60 \%$ aurait conduit à des épaisseurs de blindage telles qu'un traitement de "détensionnement » de ces derniers aurait été nécessaire; il en serait résulté, non seulement une augmentation de prix mais aussi des difficultés et des délais d'exécution supplémentaires.

M. Amrlard apporte les informations ci-après:

$1^{\circ}$ Neyrpic-Sogreah, en collaboration avec E.D.F.-R.E.H. Nord, a mis au point un programme sur ordinateur qui peut s'appliquer à tous les schémas d'installation, mais en utilisant la méthode Bergeron. Confrontés avec ceux donnés par le programme des Etudes et Recherches d'E.D.F., les résultats de ces deux modèles se révètent très comparables;

$2^{\circ}$ Il faut prendre une marge de sécurité par rapport aux résultats du calcul car on ne connaît pas la façon dont se déforment les « collines » d'essais en turbine et en pompe lorsqu'on passe du modèle au prototype industriel.

M. Simonnot fait observer que la recherche d'une réduction des investissements par la concentration des adductions, a conduit à Revin à aggraver les surpressions lors des régimes transitoires.

M. Marvaud (C.N.R.) pose les deux questions suivantes:

$1^{\circ}$ Sous quelle forme ont été entrées dans le programme les caractéristiques de la roue (analytique ou sous forme de tableau de chiffres)?

$2^{\circ}$ Comment se fait-il que l'auteur ait utilisé un schéma d'intégration numérique dont le critère de Von Neumann dit qu'il est instable?

M. Planchard répond que les caractéristiques des machines sont introduites sous forme de tableaux de chiffres, et que, moyennant un mode opératoire judicieusement choisi, le schéma d'intégration utilisé est stable (si le rapport des pas d'espace et de temps "est supérieur ou égal à la célérité des ondes).

M. ANDré, très intéressé par la communication de Mme BLANCHON, MM. Planchard et Simonnot, signale que deux incidents survenus au Service de la Production Hydraulique ont mis en évidence des surpressions atteignant cinq fois la pression de service $H$.

On a pensé, suivant un raisonnement dù à $M$. CampMas, que ces surpressions étaient dues à la formation d'une bulle de cavitation suivant le processus ci-après :

A la suite d'un incident, la fermeture totale du distributeur à partir d'une ouverture partielle conduit à une surpression supérieure à $\mathrm{H}+10 \mathrm{~m}$. A l'onde de retour sur le distributeur, étanche et à nette tendance à la fermeture, la dépression en amont du distributeur atteint $-(\mathrm{H}+10)$ et il se forme une bulle de cavitation qui est refermée à l'oscillation suivante, ce qui conduit à une surpression de $5 \mathrm{H}$.

Bien que cette hypothèse de $M$. Campmas ne soit pas entièrement démontrée, il semble qu'elle soit la seule possible car la surpression de $5 \mathrm{H}$ n'aurait pu être produite par un coup de bélier de Michaud qu'à la condition que l'ensemble du distributeur coupe le débit total dans des durées trop courtes pour être vraisemblables.

M. Smonnor signale que, contrairement à ce qui est courant pour les turbines, il peut être techniquement et économiquement préférable d'admettre pour les turbines-pompes, des vitesses de fermeture très élevées aux fortes ouvertures, et diminuant fortement au-dessous d'une ouverture moyenne, à déterminer selon le cas. Le régime transitoire consécutif à une telle fermeture « en deux temps » doit être examiné dans l'hypothèse d'une défaillance du dispositif qui ralentit la vitesse en fin de mancuvre. C'est précisément le danger d'apparition d'une poche de cavitation (Cf. fig. 8 et texte associé) qui semble limiter la promptitude maximale à adopter.

M. WeHENkEl signale qu'à l'usine de Vianden, la loi de fermeture du distributeur adoptée est linverse de celle préconisée par M. SIMONNOT; la fermeture commence à vitesse ralentie jusqu'à une certaine position du distributeur; au-delà, on augmente cette vitesse. En fait, la machine arrive presque à l'emballement à la fin de la fermeture ralentie où la majeure partie du débit est coupée, ce qui donne un premier coup de bélier; on ferme ensuite plus rapidement le distributeur.

Ce mode opératoire, remarque M. Simonnot, est utilisé par Ies Américains à Taum Sauk. Il conduit, entre autres, dans le cas d'adductions communes à plusieurs groupes, à une survitesse extrême plus importante que de raison (dans le cas d'un groupe unique sur son adduction, il faut prendre en compte de toute façon la survitesse extrême susceptible d'être atteinte à distributeur bloqué).

Nos groupes sont prévus, précise M. Werrenkel, pour pouvoir fonctionner en marche continue à la vitesse d'emballement.

M. LE GRanché intervient, ensuite en ces termes:

$1^{\circ}$ Dans votre exposé, vous avez indiqué au sujet de l'emplacement de la centrale, que le fait de la rapprocher de la descenderie du bassin supérieur, diminuerait le coût des blindages, mais par contre augmenterait le cout de l'adduction basse pression; pourriezvous nous préciser si votre étude des régime transitoires vous a permis de déterminer un optimum pour l'emplacement de la centrale?

$2^{\circ}$ Dans votre étude, avez-vous constaté une grande influence des pertes de charge et de l'allure des caractéristiques de la roue d'essai; dans ce dernier cas, avez-vous été amené à changer de roue d'essai ou tout simplement à modifier les courbes de la roue d'essai ?

$3^{\circ}$ Avez-vous utilisé votre programme comme sous-gamme de calcul des surpressions pour le programme d'E.D.F.-R.E.H. Nord de détermination des vitesses économiques d'adduction?

M. Simonnot répondant négativement sur ce dernier point, M. LE GRANCHÉ poursuit :

De ce fait, votre comparaison économique des différentes solutions possibles en prenant comme paramètre le temps de lancer du groupe et le $\Sigma L / S$ des adductions perd beaucoup de son intérêt puisque vous négligez la valeur actualisée des pertes de charge, qui est loin d'être négligeable.

Peut-être, observe M. le Président, le programme de calcul présenté est-il arrive trop tard dans le cas de l'usine de Revin, car 


\section{F. BLANCHON, J. PLANCHARD ef D. SIMONNOT}

à ce moment-là les plans d'exécution étaient en cours. Pour des projets ultérieurs, on pouma profiter de ce programme existant pour aider à dégrossir les problèmes et à prendre les décisions fondamentales.

Il faut avoir, pour répondre notamment à M. LE GRANCHÉ, quelques ordres de grandeur en tête, remarque M. Gérard. Le choix de l'adduction est d'abord conditionné par les caractéristiques générales du site, et notamment par le fait que le terrain, imperméable ou non, permet ou non l'économie des blindages. Ensuite, (mais c'est exact qu'il faut l'examiner très tôt), le problème des coups de bélier conduit à une optimisation plus fine. Par exemple à Revin, le choix d'un schéma évitant la majeure partie des blindages représente une économie de l'ordre de 15 millions (ce sera plus de 20 à Montezic). Puis, l'examen des coups de bélier a conduit à reperdre quelques millions, mais sans remettre en cause - et de loin - le choix fondamental.

M. Cavallé soulève la question de l'introduction des caractéristiques en régime permanent des machines hydrauliques dans les calculs de régimes transitoires sus-visés.

Les caractéristiques "machine » introduites dans les calculs de régimes transitoires sont quasi-stationnaires et sont obtenues comme lieu des points de fonctionnement stationnaires. Quel peut être l'ordre de grandeur des erreurs ainsi commises, notamment dans les domaines de fonctionnement anormal, turbine à l'emballement, par exemple? Existe-t-il des expériences pouvant donner des éléments de réponse?

Le problème, répond $\mathrm{M}$. CASACCI, est très complexe, mais en pratique la coupure du débit par la roue (efiet centrifuge) constitue le phénomène essentiel. Cette coupure du débit conditionnée principalement par le diamètre extérieur de la roue reste peu influencée par les différences de tracés.

M. Simonnot observe que, dans certains cas, il semble bien que la considération des seules caractéristiques stationnaires a conduit à des surpressions inféricures aux surpressions réelles du fait de « décollements » qui ne prennent naissance qu'en régime transitoire.
Les modulations de pression autour de la variation fondamentale, dit $M$. Casaccr, restent mal comnes et peuvent avoir pour cause des phénomènes de résonance complexes associés ou non à des décollements sur les aubages.

M. Remenieras se demande si, pour réduire l'intensité et amortir plus rapidement les coups de béliers qui surviennent lors de certains régimes transitoires évoqués précédemment, on pourrait utiliser le système qu'il a proposé il y a une dizaine d'années pour diminuer la célérité des ondes de pression dans les conduites en charge (*). Le système n'est efficaze que pour les coups de béliers « rapides » suivant la terminologie d'Allievi; mais l'intensité de ces derniers peut facilement être divisée par 10 grâce à l'introduction d'un dispositif simple augmentant la compressibilité de l'ensemble « conduite + eau ». Ce dispositif peut être réalisé de façon très robuste; ainsi dans son application (réussie) à la réduction des fortes vibrations (*) constatées dans laspirateur de grosses turbines, il était constitué par un volume dair emprisonné dans la partie haute de la cloison médiane creuse (et spécialement aménagée) dudit aspirateur. La consommation d'air est, en principe, très faible et du même ordre que celle constatée sur les réservoirs d'air antibéliers classiques dans certaines stations de pompage.

M. Srmonnot répond qu'à sa connaissance un dispositif industriel, réalisant cette élasticité sous forme linéique, n'existe pas sur le marché. L'introduction d'un débit d'air permanent dans l'eau en écoulement pourrait être envisagée. Mais le danger d'accumulation de l'air en certains endroits est important : la présence de « poches d'air » imprévues peut modifier considérablement le déroulement des régimes transitoires.

M. le Président clôt la discussion en remerciant tous ceux qui ont contribué à l'animer et donne la parole à M. INDri pour l'exposé de sa communication.

$\left(^{*}\right)$ Voir La Honille Blanche, No spécial A/1952, pp. 172-196. (**) Ces vibrations avaient probablement pour origine les a torches émises a certaines charges par la roue de la turbine.

\section{$-\ldots$}

This report deals with the problems with which the designer of pumped storage schemes with reversible sets is faced when he considers the effect of unsteady machine regimes on the project. The various factors which enter into scheme design are briefly reviewed. These are:

(a) The characteristic times of the conduits (examples of which are given in Figure 1) and the economic gains which result from concentrating the supply ducts.

(b) The very important problem of shutting off the flow through the turbines. The sequence of operating regimes is briefly described (these may include temporary flow inversion). It is shown that shutting down quickly in two short periods may often be preferable since this puts a limit on excess pressures attained and also avoids secondary phenomena. The effect of machine moment of inertia and of the surge tank selected is discussed in the light of scheme site characteristics.

(c) Unsteady regimes arising from loss of driving torque in pumpwise operation are examined, together with several purely accidental regimes. For instance, Figure 8 shows initiation of water hammer arising out of very rapid guide-vane closure.
The example shown in Figure 9 presents the results of an economic optimization study in terms of the unsteady regimes obtained and the important variables (run-up time of reversible power set and supply ducting). Here the overpressures were calculated by a simple method, wave celerities being infinite. Figure 10 justifies this approximation

For more accurate calculation of the excess pressures and speeds to be considered in projects and project guarantees, a more comprehensive method should be used.

Electricité de France has written a computer programme to determine these unsteady regimes. A brief description of the programme is given in the appendix, together with a list of the simplifying assumptions. The programme permits calculation of flows, pressures and unit speeds at any moment and at any point in a hydraulic system comprised of basins, feed conduits, turbine-pumps with speed governors, surge tanks ctc., for all kinds of system structure.

The solution is obtained by applying a difference scheme to the Alliévi equations. 